\title{
HYDROCELE OF THE CANAL OF NUCK: A RARE DEVELOPMENTAL ANOMALY
}

Mathangasinghe $\mathrm{Y}^{1}$, Kalubowila $\mathrm{JN}^{2}$

${ }^{1}$ Department of Anatomy, Faculty of Medicine, University of Colombo, Sri Lanka.

${ }^{2}$ Provincial General Hospital, Ratnapura, Sri Lanka.

DOI: http://doi.org/10.4038/sljr.v4i1.75

Keywords: Canal of Nuck, Hydrocele

\section{Introduction}

Hydrocele of the Canal of Nuck is a developmental abnormality in females characterized by accumulation of fluid in a patent peritoneal extension along the round ligament of the uterus. Approximately 400 cases of this entity have been reported worldwide $^{1}$. This has no been reported previously in Sri Lanka.

\section{Case Report}

A 42-year-old female presented with a lump in the right groin of two months duration. On examination, it was an irreducible cystic lump with mild tenderness. There was no expansile cough impulse. Computed Tomography (CT) with intravenous, oral and rectal contrast demonstrated a welldefined oval shaped fluid density lesion (12 Hounsfield Units) in the right groin measuring $4 \times 4 \times 6 \mathrm{~cm}$ with reactive inguinal lymphadenopathy (Figure 1 and 2). There was no solid component. The fluid collection did not communicate with the peritoneal cavity. Appearance was in favour of a hydrocele of the Canal of Nuck. There was no evidence of inguinal hernia. Patient underwent open hydrocelectomy and high ligation. Postoperative period was unremarkable. Patient was asymptomatic after three months follow up.

\section{Discussion}

\section{Anatomy and embryology}

During the development in females, the round ligament of the uterus drags a peritoneal fold with it as it descends to the labium majus through the inguinal canal. This peritoneal elongation is known as the Canal of Nuck. It is the female counterpart of the processus vaginalis in males. The Canal of Nuck obliterates during fetal life ${ }^{2}$

Corresponding Author: Yasith Mathangasinghe <yasith@anat.cmb.ac.lk> 
or within the first year of life ${ }^{3}$. Persistence of this communication within the inguinal canal predisposes to an indirect inguinal hernia whereas partial obliteration leads to the development of a hydrocele of the Canal of $\mathrm{Nuck}^{1,4-6}$. Our patient had an isolated hydrocele of the Canal of Nuck with no evidence of an indirect inguinal hernia.

\section{Clinical features}

Classically the hydrocele of the Canal of Nuck is described as an asymptomatic lump ${ }^{1,7}$. Patient may give a history of a lump first appearing at the superficial inguinal ring and progressively descending to labia majora ${ }^{4}$. Examination usually reveals a reducible cystic translucent lump in the inguinal region ${ }^{7}$. Presence of cough impulse and bowel sounds over the lump is suggestive of a hernia ${ }^{1,4,7}$. But clinically distinguishing an indirect inguinal hernia from a hydrocele of the Canal of Nuck is difficult and may not be accurate ${ }^{4}$. Both a hernia and a hydrocele may coexist ${ }^{4,8}$. Hydrocele of Canal of Nuck is often mistaken clinically for incarcerated inguinal herniae leading to unnecessary emergency laparotomies ${ }^{9}$. The lump of our patient was irreducible, but the presence of fluctuation and trans-illumination with no cough impulse were characteristic for hydroceles of the Canal of Nuck.

\section{Investigation}

Characteristic imaging findings to accurately diagnose hydrocele of Canal of Nuck are described scarcely ${ }^{6}$. It is visualized as a well circumscribed thin walled fluid collection along the pathway of inguinal canal in both ultrasound and CT imaging ${ }^{1}$. Unilocular appearance is commoner than multilocular appearance ${ }^{6}$. Internal septations may or may not be seen on imaging ${ }^{1}$. Presence of a soft tissue component, thick wall, irregular wall, calcifications or increased vascularity in the vicinity are suggestive of alternative diagnoses $^{6,10}$. In our case we could demonstrate a well-defined oval shaped fluid density lesion in the inguinal region.

\section{Treatment and prognosis}

The management of a hydrocele of the Canal of Nuck depends on the symptoms ${ }^{1,6}$. Direct or ultrasound guided aspiration may rapidly relieve symptoms. Aspiration of the cystic fluid is associated with a high incidence of recurrence ${ }^{1}$. Therefore, hydrocelectomy and high ligation is the gold standard of treatment ${ }^{5,10}$. Minimally invasive laparoscopic surgeries are increasingly performed with good surgical outcomes ${ }^{2}$. In 
our case an open hydrocelectomy a high ligation was successfully performed.

\section{Conclusions}

Hydrocele of the canal of Nuck is a rare benign developmental abnormality seen in females. It is characterized by accumulation of fluid in a patent peritoneal extension along the round ligament of the uterus which appears as a cystic lump in the inguinal canal or labia majora. Ultrasound and CT imaging show a thin walled fluid collection in the pathway of inguinal canal. Presence of a soft tissue component, thick wall or calcifications suggest alternative diagnoses. It can be managed either conservatively or surgically depending on the symptoms. Minimally invasive laparoscopic surgeries are increasingly performed with good surgical outcomes.

\section{Acknowledgements}

Authors thank Dr. Udari Apsara Liyanage, Consultant Radiologist and Senior Lecturer in the Department of Anatomy, Faculty of Medicine, University of Colombo.

\section{Conflicts of interests}

Authors declare that there are no conflicts of interests

\section{Reference}

1. Sethi, V. and H. Patel, Hydrocele in the Canal of Nuck-CT appearance of a developmental groin anomaly. Journal of radiology case reports, 2016. 10(11): p. 29.

2. Matsumoto, T., et al., Laparoscopic diagnosis and treatment of a hydrocele of the canal of Nuck extending in the retroperitoneal space: A case report. International journal of surgery case reports, 2014. 5(11): p. 861-864.

3. Park, S., et al., Hydrocele of the canal of Nuck in a girl: ultrasound and MR appearance. The British journal of radiology, 2004. 77(915): p. 243-244.

4. Counseller, V.S. and B.M. Black, Hydrocele of the canal of Nuck: report of seventeen cases. Annals of surgery, 1941. 113(4): p. 625.

5. Mandhan, P., Z. Raouf, and K. Bhatti, Infected hydrocele of the Canal of Nuck. Case reports in urology, 2013. 2013.

6. Stickel, W.H. and M. Manner, Female hydrocele (cyst of the canal of Nuck). Journal of ultrasound in medicine, 2004. 23(3): p. 429-432.

7. De Meulder, F., et al., Female hydrocele of the canal of Nuck: a case report. European journal of pediatrics, 2006. 165(3): p. 193-194. 
8. Keen, W.W. and J.C. Da Costa, Surgery, its principles and practice. Vol. 4. 1914: WB Saunders.

9. Yen, C.-F., et al., Laparoscopic closure of patent canal of Nuck for female indirect inguinal hernia. The Journal of the American Association of Gynecologic Laparoscopists, 2001. 8(1): p. 143-146.

10. Jagdale, R., et al., Hydrocele of the canal of Nuck: value of radiological diagnosis. Journal of radiology case reports, 2012. 6(6): p. 18.
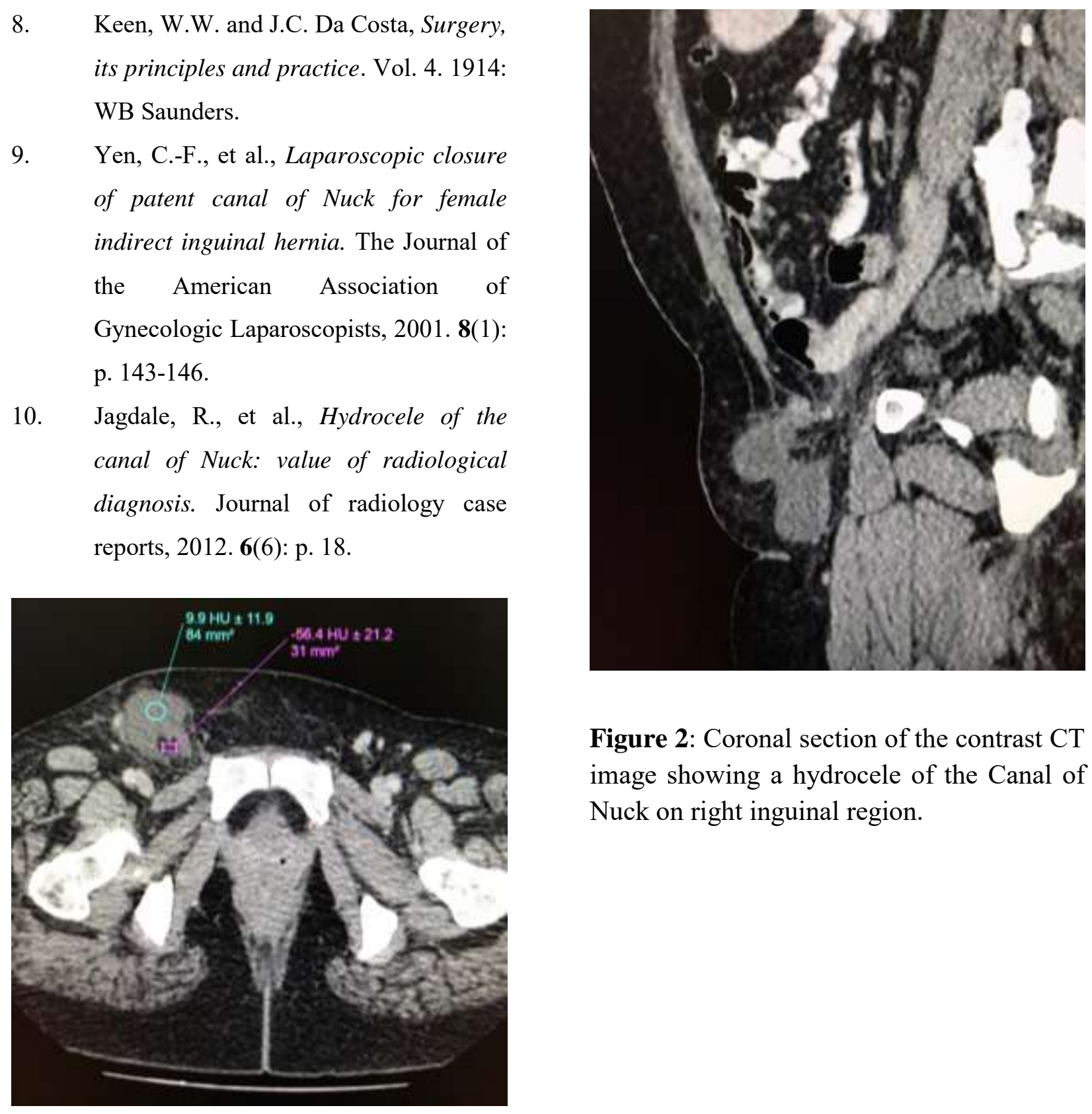

Figure 2: Coronal section of the contrast CT image showing a hydrocele of the Canal of Nuck on right inguinal region.

Figure 1: Axial section of the contrast CT image showing a hydrocele of the Canal of Nuck on right inguinal region. (HU Hounsfield Units) 